JOURNAL CLUB

\title{
Riociguat, a novel therapy for pulmonary hypertension?
}

This phase three, double-blind, randomised, placebo-controlled, multicentre study was carried out in 30 countries. It compared riociguat (maximum dose $2.5 \mathrm{mg}$ three times a day) with placebo in the treatment of symptomatic pulmonary hypertension.

The primary end point was change in baseline walking distance over 6 min assessed after 12 weeks of treatment. Patients in the riociguat group improved by a mean $30 \mathrm{~m}$ increase compared with a mean $6 \mathrm{~m}$ decrease in the placebo group (least-squares mean difference $36 \mathrm{~m}$; 95\% CI 20 to $52 ; \mathrm{p}<0.001$ ).

Secondary end points included: change in pulmonary vascular resistance; N-terminal probrain natiuretic peptide levels; WHO functional class; time to clinical worsening; Borg dyspnoea scale score; quality of life; safety. Patients on riociguat showed significant improvements throughout. Gastrointestinal symptoms occurred more often with riociguat.

Although not powered for subgroup analyses, improvements occurred in patients on background therapy plus riociguat and riociguat alone. Greatest benefits were seen in WHO functional classes III and IV. Patients on phosphodiesterase type 5 inhibitors, intravenous prostanoids and those with pulmonary hypertension secondary to HIV, schistosomiasis and chronic haemolytic anaemia were excluded. Care must be taken when extrapolating data to the wider population with pulmonary hypertension.

The magnitude of the trial's primary end point was at the lower end of that expected by the authors, perhaps because some patients were already on standard therapies and had better than expected WHO functional class. However, baseline patient characteristics were balanced across the study arms and the percentage dropout was similar. This study supports the use of riociguat as a novel therapy in symptomatic pulmonary hypertension. Longer-term safety and efficacy data collection is ongoing.

- Ghofrani H, Galiè N, Grimminger F, et al. Riociguat for the treatment of pulmonary arterial hypertension. N Eng/ J Med 2013;369:330-40.

Sarah-Jane Bailey

Correspondence to Dr Sarah-Jane Bailey, CT2, University Hospital Lewisham NHS Trust, Department of Respiratory Medicine, Lewisham High Street, London SE13 6LH, UK; sarah-jane.bailey@nhs.net

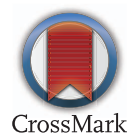

To cite Bailey S-J. Thorax 2014;69:1154.

Thorax 2014;69:1154. doi:10.1136/thoraxjnl-2013-204664 